\title{
ANDES

www.scielo.cl

\section{Estimación de la prevalencia de trastorno del Espectro Autista en población urbana chilena}

\author{
Prevalence estimation of Autism Spectrum disorders in chilean urban population
}

\author{
Carolina Yáñez ${ }^{\mathrm{a}}$, Paulina Maira ${ }^{\mathrm{b}}$, Constanza Elgueta ${ }^{\mathrm{b}, \mathrm{c}}$, Macarena Brito ${ }^{\mathrm{d}}$, Marcelo A. Crockett $\mathrm{t}^{\mathrm{e}}$, \\ Ledia Troncoso $^{\text {a }}$, Claudia López ${ }^{\mathrm{a}}$, Mónica Troncoso ${ }^{\mathrm{a}}$
}

\author{
aServicio de Neuropsiquiatría Infantil, Hospital Clínico San Borja Arriarán. Santiago, Chile \\ buniversidad de Chile. Santiago, Chile \\ 'Facultad de Medicina. Universidad de Chile. Santiago, Chile \\ dResidente Psiquiatría Infanto Juvenil. Escuela de Medicina. Pontificia Universidad Católica de Chile. Santiago, Chile. \\ ePsicólogo. ANID, Programa Iniciativa Científica Milenio, Núcleo Milenio para Mejorar la Salud Mental de Adolescentes y Jóvenes (Imhay). \\ Santiago, Chile. Escuela de Salud Pública, Universidad de Chile, Santiago, Chile
}

Recibido: 14 de mayo de 2020; Aceptado: 4 de enero de 2021

¿Qué se sabe del tema que trata este estudio?

La prevalencia de Trastorno del Espectro Autista ha ido en aumento. La importancia de monitorizar esta condición está dada por la necesidad de determinar e implementar recursos necesarios para el abordaje terapéutico de estos pacientes en cada comunidad. No se conoce la prevalencia chilena de Trastorno del Espectro Autista.
¿Qué aporta este estudio a lo ya conocido?

Se calculó la prevalencia de Trastorno del Espectro Autista en una muestra urbana de niños entre 18-30 meses de dos comunas urbanas de la Región Metropolitana de Santiago, Chile. Se obtuvo una prevalencia de 1 en 51 niños, con una distribución por sexo de 4 niños por 1 niña.

\section{Resumen}

La prevalencia del Trastorno del Espectro Autista (TEA) ha aumentado, variando entre 0,5 y $1 \%$ alrededor del mundo. Se desconoce la prevalencia de TEA en Chile. Objetivo: Estimar la prevalencia de Trastorno del Espectro Autista en 2 comunas urbanas de Santiago de Chile. Sujetos y Método: Estudio epidemiológico transversal. Participaron 272 niños entre 18-30 meses que asistían a control sano en dos Centros de Salud Familiar de dos comunas urbanas de la capital. Se utilizó un muestreo consecutivo y se excluyeron los niños que ya estaban siendo controlados por neurología. Se realizó un tamizaje mediante el Cuestionario de Autismo en Niños - Modificado (MCHAT). Los niños con MCHAT alterado fueron evaluados por neuropediatra en el Hospital Clínico San Borja Arriarán y diagnosticados con Trastorno del Espectro Autista de acuerdo a criterio clínico. Se administró la Escala de Observación para el Diagnóstico del Autismo - Segunda Versión (ADOS-2) como complemento diagnóstico. Se estimó la prevalencia de Trastorno del Espectro Autista con intervalo de confianza al
Palabras clave:

Prevalencia;

Trastorno del Espectro Autista;

Autismo;

Detección Temprana

Correspondencia:

Carolina Yáñez

cyanez.uchile@gmail.com 
95\%. Resultados: 44 niños tuvieron M-CHAT alterado; de ellos 5 fueron diagnosticados clínicamente con Trastorno del Espectro Autista. Se obtuvo una prevalencia de Trastorno del Espectro Autista de 1,95\% (IC95\% 0,81-4,63), con una distribución por sexo de 4 niños por 1 niña. Conclusiones: Esta es la primera estimación de prevalencia de Trastorno del Espectro Autista en dos comunas de Santiago, Chile. Se obtuvo una alta prevalencia de esta condición, lo que deja en evidencia la necesidad de obtener recursos para el abordaje multidisciplinario precoz de estos pacientes.

\section{Abstract}

The prevalence of Autism Spectrum Disorder has increased, varying between 0.5 and $1 \%$ around the world. The prevalence of ASD in Chile is unknown. Objective: To estimate the prevalence of ASD in two urban communes of Santiago, Chile. Subjects and Method: Cross-sectional epidemiological study. 272 children aged between 18-30 months who attended well-child visits at two Family Health Centers in two urban communes of Santiago participated. Consecutive sampling was used and children who were already being monitored by neurology were excluded. Screening was performed using the Modified Checklist for Autism in Toddlers (M-CHAT). Those children with altered M-CHAT were evaluated by a pediatric neurologist at the San Borja Arriarán Clinical Hospital and diagnosed with ASD according to clinical criteria. The Autism Diagnostic Observation Schedule - Second Version (ADOS-2) was used as a diagnostic complement. The prevalence of ASD was estimated with a 95\% confidence interval. Results: 44 children had altered M-CHAT; 5 of them were clinically diagnosed with ASD. A 1.95\% prevalence of ASD (95\% CI 0.81-4.63) was obtained, with a sex distribution of 4 boys per 1 girl. Conclusions: This study is the first estimate of ASD prevalence in two communes of Santiago, Chile. A high prevalence of this condition was observed, which highlights the need for obtaining resources for an early multidisciplinary approach for these patients.

\section{Keywords:}

Prevalence;

Autism Spectrum

Disorder;

Autism;

Early Detection

\section{Introducción}

Los Trastornos del Espectro Autista (TEA) son un grupo de alteraciones clínicas que incluyen deficiencias persistentes en la comunicación e interacción social en diversos contextos, además de patrones restrictivos y repetitivos de comportamiento, intereses o actividades, causando deterioro clínico significativo en el área social, laboral u otras importantes para el funcionamiento habitual ${ }^{1}$. La prevalencia de TEA ha ido en aumento continuo y estable en los últimos 30 años $^{2}$, fluctuando en la actualidad entre el 0,5 y $1 \%$ alrededor del mun$\mathrm{do}^{3-5}$. Según el Centers for Disease Control and Prevention (CDC) el año 2014 la prevalencia en Estados Unidos llegaba a 1 en 59 niños de 8 años $^{3}$. Las causas de este aumento de prevalencia son controversiales y siguen siendo motivo de estudio. Se han atribuido a la heterogeneidad de los pacientes incluidos en las muestras, la falta de metodología estandarizada para realizar estudios epidemiológicos en TEA y a un aumento real dado por factores ambientales que han sido difíciles de identificar ${ }^{2,6}$.

La importancia de monitorizar este cuadro se ha vuelto una prioridad en salud pública en muchos países $^{2}$. Esta información proporciona valiosos beneficios, constituyendo un catastro de los servicios existentes y ayudando a evaluar las necesidades y prioridades de cada comunidad ${ }^{4}$. Obtener una prevalencia local de
TEA podría ayudar a determinar el verdadero déficit de recursos terapéuticos para estos pacientes y lograr su implementación.

Se han realizado estudios de prevalencia de TEA en Venezuela, Brasil y Argentina, pero sus metodologías han sido cuestionadas ${ }^{2}$ y actualmente se desconoce la prevalencia chilena.

El objetivo de este trabajo fue estimar la prevalencia de TEA en niños y niñas entre 18-30 meses en centros de atención primaria de salud urbanos.

\section{Sujetos y Método}

Se realizó un estudio transversal descriptivo.

\section{Participantes}

La población en estudio corresponde a niños y niñas entre 18 y 30 meses que se atienden en el sistema público de salud, residentes de las comunas de Estación Central y Santiago Centro de la Región Metropolitana de Santiago, Chile correspondientes a una parte de la población evaluada en el Servicio de Neuropsiquiatría Infantil del Hospital Clínico San Borja Arriarán (HCSBA).

El cálculo de tamaño muestral se realizó mediante la fórmula para poblaciones finitas considerando: una prevalencia de $0,5^{7}$, el tamaño de la población para este 
rango de edad según el Censo 2012 (correspondiente a 8.366 niños $)^{8}$, un nivel de confianza de $90 \%$ y una precisión del 5\%. El tamaño de muestra a evaluar fue de 264 niños.

Se realizó un muestreo consecutivo. Los criterios de inclusión fueron: 1) niños que asistían a control sano o consulta de morbilidad no neurológica en los Centros de Salud Familiar (CESFAM) de Chuchunco (Estación Central) y CESFAM Nº1 Ramón Corbalán Melgarejo (Santiago Centro) y 2) que tuvieran entre 18 y 30 meses de edad. De acuerdo con las bases de datos de los CESFAM participantes, éstos atienden principalmente familias de nivel socioeconómico bajo y medio, usuarios mayoritariamente del Fondo Nacional de Salud (FONASA).

Se utilizaron datos del Censo del año 2012, por ser el único disponible al iniciar el estudio.

Por otra parte, se excluyeron los niños que ya estaban siendo controlados en el servicio de neuropsiquiatría infantil del HCSBA debido a que habían sido evaluados mediante procedimientos diferentes a los propuestos en esta investigación. La recolección de la muestra se realizó entre agosto 2016 y agosto 2018.

\section{Medidas}

\section{Cuestionario de Autismo en la Infancia Modificado $(\mathrm{M}-\mathrm{CHAT})^{9}$}

Prueba de cribado para la detección de TEA en niños. Se utilizó la versión española del cuestionario que posee 23 ítems con un formato de respuesta dicotómico $(1=n o, 0=s i)$. Se consideró como resultado alterado quienes respondieron no a 3 o más ítems o que respondieron no para 2 o más ítems críticos (ítems 2, $7,9,13,14$ y 15$)$.

\section{Autism Diagnostic Observation Schedule - second edition (ADOS-2)}

Escala de Observación para el Diagnóstico de Autismo-segunda edición ${ }^{10,11}$. Evaluación observacional estandarizada y semiestructurada para síntomas de TEA, compuesta por 5 módulos que se utilizan según la edad cronológica y el nivel de lenguaje del niño/a. En este estudio se utilizó la versión española del instrumento y se administraron los dos primeros módulos ( T y 1) como complemento de la evaluación clínica, por ser este instrumento considerado gold standard con fines de investigación ${ }^{12}$.

\section{Trastorno de Espectro Autista}

Se diagnosticaron con TEA a los niños que cumplieron con los criterios diagnósticos clínicos según el Manual Diagnóstico y Estadístico de los Trastornos Mentales, quinta edición (DSM-5), de acuerdo con la evaluación de una neuróloga infantil del servicio de neuropsiquiatría Infantil del HCSBA.

\section{Procedimiento}

El estudio fue aprobado por el Comité de Ética del Servicio de Salud Metropolitano Central. Enfermeras y médicos generales, pediatras y neurólogos infantiles, previamente capacitados, encuestaron con $\mathrm{M}^{-\mathrm{CHAT}^{9}}$ a cuidadores de los niños que cumplieran los criterios de inclusión y que dieran su consentimiento informado. Los niños con M-CHAT alterado fueron citados a evaluación por neuróloga infantil al policlínico de Trastornos del Neurodesarrollo en el HCSBA y se aplicó ADOS-2 por profesionales capacitados en el instrumento, como complemento del diagnóstico clínico. Los niños posteriormente fueron ingresados a control y manejo por equipo multidisciplinario en el mismo centro asistencial.

\section{Análisis estadístico}

Se estimó la prevalencia con intervalo de confianza al 95\% (IC95\%) de los niños que fueron diagnosticados con TEA. Dado que hubo participantes con M-CHAT alterado que no pudieron ser evaluados clínicamente, se realizó un análisis de sensibilidad ${ }^{13}$, el cual tiene por objetivo examinar los resultados bajo determinados supuestos, como una aproximación a la evaluación del error sistemático, que en el estudio podría estar dado por la pérdida del seguimiento de estos participantes. Para ello se imputaron los datos de los participantes que no completaron la evaluación clínica bajo tres supuestos: 1) todos los casos tenían TEA, 2) todos los casos no tenían TEA, 3) los casos se distribuyeron con la misma proporción de casos con TEA positivos de quienes sí fueron evaluados clínicamente. Para cada supuesto se estimó la prevalencia e IC95\%.

\section{Resultados}

La figura 1 muestra el flujograma del proceso de evaluación de los participantes. Se administró el MCHAT a 300 niños que acudieron a control sano o consulta de morbilidad. De ellos, 20 fueron excluidos por falta de datos y 8 rechazaron participar en el estudio. Fueron incluidos en total 272 niños, de los cuales 146 son niños $(53,7 \%)$ y 126 niñas $(46,3 \%)$. La edad media de los participantes fue de 22,2 meses (desviación estándar 3,7 meses).

Del total de participantes, 44 (16\%) tuvieron MCHAT alterados, quienes fueron citados al servicio de neuropsiquiatría infantil HCSBA para evaluación clínica por neuróloga infantil. De los 44 niños con MCHAT alterado, se evaluaron 28 y 16 de los pacientes evaluados con M-CHAT alterado se perdieron del seguimiento y no lograron la evaluación clínica por el especialista (no acudieron a la citación o no lograron ser contactados). 
Figura 1. Flujograma del proceso de evaluación de los participantes.

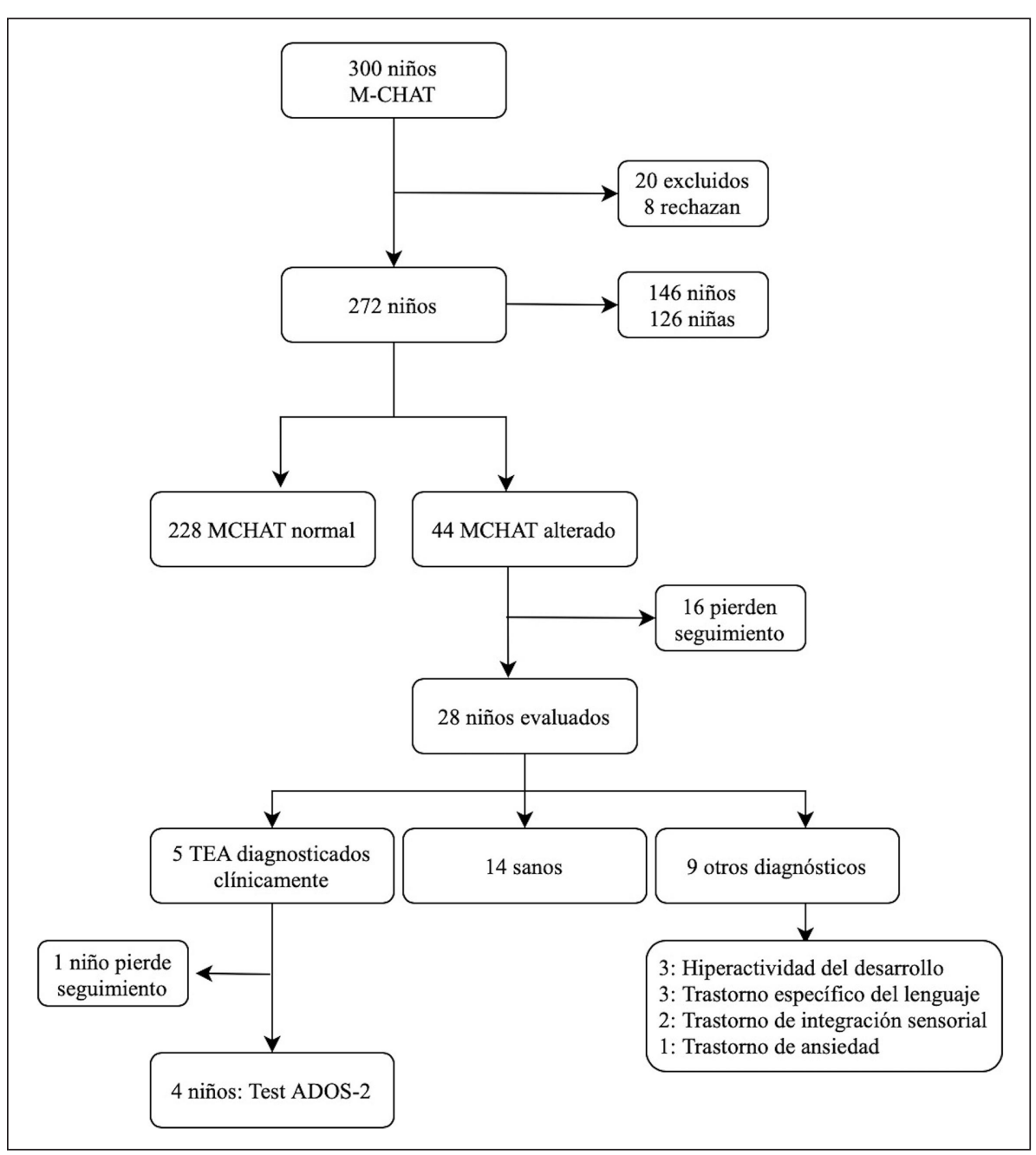

De los 28 niños evaluados, 5 fueron diagnosticados clínicamente como TEA, 14 se encontraban sanos y 9 presentaron otros diagnósticos: 3 hiperactividad del desarrollo, 3 trastorno específico del lenguaje, 2 trastorno integración sensorial y 1 trastorno de ansiedad.

De los 5 niños clínicamente diagnosticados con TEA, 4 fueron evaluados con ADOS-2 y 1 se perdió en el seguimiento. Respecto a la aplicación de ADOS-2, a 3 niños se les evaluó con el módulo T, encontrándose todos en rango de preocupación moderado a severo, y a 1 se le aplicó módulo 1, con resultado en rango de
TEA moderado, apoyando de esta forma el diagnóstico clínico. Como se mencionó, sólo a 4 de los 5 niños se les hizo test de ADOS-2. Tomando en cuenta que el diagnóstico de TEA es clínico se consideraron a los 5 pacientes con este cuadro. Finalmente, de los pacientes diagnosticados con TEA, 4 fueron niños y 1 niña.

La prevalencia obtenida en la muestra y bajo los supuestos del análisis de sensibilidad están en la tabla 1. La prevalencia de TEA en la muestra final $(n=256)$ fue de 1,95\% (IC95\% 0,81-4,63). El porcentaje de niños con TEA entre quienes tenían M-CHAT alterados y

\section{Tabla 1. Prevalencia de Trastorno del Espectro Autista (TEA) y análisis de sensibilidad}

\begin{tabular}{|c|c|c|c|}
\hline & & Prevalencia & IC $95 \%$ \\
\hline Muestra total con información completa & $(n=256)$ & 1,95 & $0,81-4,63$ \\
\hline Supuesto 1: 16 casos perdidos con TEA & $(n=272)$ & 7,72 & $5,07-11,58$ \\
\hline Supuesto 2: 16 casos perdidos sin TEA & $(n=272)$ & 1,84 & $0,76-4,36$ \\
\hline Supuesto 3: 16 casos perdidos con misma proporción de TEA de casos evaluados (3 con TEA de 16) & $(n=272)$ & 2,94 & $1,47-5,79$ \\
\hline
\end{tabular}


que fueron evaluados clínicamente fue de 17,9\%. Según este porcentaje se imputaron los datos de los 16 casos perdidos en el seguimiento para el supuesto $3 \mathrm{del}$ análisis de sensibilidad, es decir, se imputaron 3 casos como TEA y 13 como sin TEA. El análisis de sensibilidad muestra que, a excepción del supuesto 1 que es el menos probable ya que se consideran los 16 casos perdidos como TEA, la prevalencia bajo determinados supuestos no sería estadísticamente diferente de la estimada, ya que los intervalos de confianza se superponen.

\section{Discusión}

La prevalencia de TEA obtenida en la muestra fue de 1,96\% (IC95\% 0,81-4,63), es decir, 1 en 51 niños, con una distribución por sexo de 4 niños por 1 niña. Esta prevalencia se asemeja a las más altas reportadas a nivel mundial, con predominio en el sexo masculino, lo que se alinea con lo reportado en la literatura ${ }^{3}$. La elevada prevalencia obtenida en el trabajo, si bien es similar a la reportada por el Center for Disease Control (CDC) de Estados Unidos, puede deberse a la edad de los niños, ya que a menor edad los síntomas no persisten en todos, sino sólo en un 85 a 90\% de ellos; a diferencia de lo que ocurre con el diagnóstico de TEA a mayor edad que es más estable. Por otra parte, no se registraron datos de factores ambientales, los cuales aumentan la prevalencia de TEA y podrían explicar la cifra obtenida, tales como: edad de ambos padres, patología del embarazo, prematurez, entre otros ${ }^{14,15}$.

Respecto a la estimación de prevalencia, las diferencias de ésta entre los estudios son difíciles de evaluar y cuesta determinar si las discrepancias observadas se deben a factores del método o verdaderas diferencias en los parámetros de la población ${ }^{16}$. Las limitaciones de la falta de un método estandarizado para llevar a cabo estudios epidemiológicos incluyen: el rango de edad de los participantes, diferentes tamaños muestrales, criterios diagnósticos utilizados, entre otros ${ }^{2}$. Respecto a los instrumentos de pesquisa, la mayoría se basa en la historia clínica entregada por los padres 5 . El CDC ha realizado tres tipos de recolección de datos: encuestas a los padres sobre diagnóstico de TEA a lo largo de la vida de los niños, expertos clínicos que revisan los registros médicos y educacionales de los pacientes y encuestas respondidas por padres reportando sobre diagnóstico actual de TEA. Esta metodología tiene múltiples limitaciones como sesgo de recuerdo y falta de seguimiento de los pacientes. Las cifras de prevalencia reportadas por el CDC son de las más altas fluctuando entre un 1,47 a 2,2\% según la metodología empleada ${ }^{3}$. De esta forma, algunos estudios utilizan instrumentos para identificar casos, enfocándose en niños previamente diagnosticados con TEA o alguna otra alteración del neurodesarrollo. Esta aproximación no permite a los investigadores detectar pacientes sin una condición previamente identificada y resulta en una desconocida, y probablemente imperfecta sensibilidad. Por el contrario, en el presente estudio se realizó una evaluación en población neurológicamente sana.

La prevalencia de TEA ha sido ampliamente estudiada en países en desarrollo, a diferencia de lo que ocurre en el resto del mundo donde la disponibilidad de estos datos es limitada. Existen estimaciones en México y en algunos países sudamericanos. En un estudio realizado en Guanajuato, se determinó una prevalencia de $0,87 \%$ de TEA en niños de 8 años 2 . En Venezuela se reportó una prevalencia de $0,17 \%$ de TEA en niños de 3-9 años ${ }^{17}$; mientras que en Argentina mostró una prevalencia de 1,3\%, difícil de interpretar dado su metodología ${ }^{18}$. En Brasil una estimación preliminar de 0,27 $\%$ fue reportada con una muestra de 1.470 niños de 7 a 12 años en el distrito de Atibaia ${ }^{19}$.

Un trabajo realizado en Canarias, España ${ }^{20}$, de metodología similar a la utilizada en este estudio, obtuvo una prevalencia de $0,61 \%$. En él se descartaron falsos positivos a través de llamada telefónica de seguimiento, que corroboraba las respuestas iniciales. En el trabajo español se analizaron los resultados por tramo de edad evidenciando el mayor número de falsos positivos en los más pequeños.

Una de las principales implicancias de esta investigación radica en que, estimar la prevalencia local de TEA podría ayudar a la toma de decisiones sobre políticas públicas en salud y educación, permitiendo orientar la planificación y distribución de recursos en estos ámbitos, que favorezcan el abordaje multidisciplinario de niños y niñas con TEA. La alta prevalencia encontrada da cuenta de que el TEA podría ser considerado como un problema de salud pública.

Existen estudios de prevalencia que tratan de identificar niños TEA en el contexto escolar ${ }^{2}$. En este trabajo, se realizó el reclutamiento en atención primaria de salud, pues la escolarización a la edad de los niños incluidos en esta investigación no es obligatoria en Chile. Dentro del flujograma diagnóstico, los controles de salud en atención primaria constituyen una buena puerta de entrada, considerando la cobertura que tienen en nuestro país ${ }^{21}$. Se eligió una muestra del sistema de salud público chileno, porque éste acoge a la mayor parte de la población (en torno al $80 \%$ de niños menores de 10 años), brindando una buena cobertura de los controles de salud $\mathrm{d}^{22}$.

Una de las principales limitaciones del trabajo es que, además de la edad y el sexo de los pacientes, no se registraron otros datos sociodemográficos ni médicos de los participantes.

No se consideraron pacientes con patología pre- 
via. El no haber considerado a estos pacientes ni sus características, puede haber incidido en una menor prevalencia obtenida, lo que consideramos como una limitación de nuestro estudio.

Otra limitación es que se utilizaron las versiones españolas de los dos instrumentos utilizados, debido a que en el momento del estudio no se contaba con las adaptaciones a la población chilena. Posteriormente se publicó la adaptación chilena del M-CHAT R/F (Revised with Follow-up $)^{12}$, por lo que sería importante incluir esta versión del instrumento en futuros estudios sobre TEA en este rango de edad.

Otra de las limitaciones de este estudio corresponde a la pérdida de seguimiento de 16 casos. Su pérdida pudo haber afectado la estimación de la prevalencia, dado que son casos que tuvieron la prueba de screening (M-CHAT) positiva. En este sentido, para evaluar el posible impacto de esta pérdida de casos se realizó el análisis de sensibilidad imputando los datos faltantes de estos casos bajo determinados supuestos. Según este análisis, los resultados no mostraron diferencias importantes entre la prevalencia sin los 16 casos y con los 16 casos imputados bajo determinados supuestos, a excepción del primer escenario, que corresponde al menos probable ya que los 16 casos se imputaron como TEA.

Por otro lado, otra posible limitación es que no se realizó una evaluación clínica de los participantes con M-CHAT negativo para buscar falsos negativos. En este trabajo se utilizó un diseño transversal, por lo que no se realizó un seguimiento a los participantes. Es recomendable en investigaciones futuras utilizar diseños longitudinales para explorar la persistencia de los síntomas de TEA.

Las fortalezas radican en que se administró la prueba de cribado M-CHAT por personal capacitado, además de que se realizó un diagnóstico clínico por neuróloga infantil, subespecialista en Trastornos del Neurodesarrollo. Sumado a esto, como apoyo al diagnóstico clínico se administró ADOS-2 por personal entrenado, siendo considerado este instrumento como gold standard en investigación ${ }^{12}$. Los resultados de dicha prueba fueron concordantes con los diagnósticos clínicos.

\section{Conclusiones}

La prevalencia de TEA obtenida en la muestra fue de 1,96\% (IC95\% 0,81-4,63), es decir, 1 en 51 niños, con una distribución por sexo de 4 niños por 1 niña.
Este estudio proporciona evidencia de que en una zona urbana de Chile habría una alta prevalencia de esta condición, tal como ocurre en el resto del mundo, lo que la convierte en un problema de salud, con la inminente necesidad de tomar decisiones de políticas públicas en educación y salud para el estudio y abordaje multidisciplinario de estos pacientes y así responder a las necesidades de los niños chilenos con TEA.

\section{Responsabilidades Éticas}

Protección de personas y animales: Los autores declaran que los procedimientos seguidos se conformaron a las normas éticas del comité de experimentación humana responsable y de acuerdo con la Asociación Médica Mundial y la Declaración de Helsinki.

Confidencialidad de los datos: Los autores declaran que han seguido los protocolos de su centro de trabajo sobre la publicación de datos de pacientes.

Derecho a la privacidad y consentimiento informado: Los autores han obtenido el consentimiento informado de los pacientes y/o sujetos referidos en el artículo. Este documento obra en poder del autor de correspondencia.

\section{Agradecimientos}

Queremos expresar nuestro agradecimiento a la doctora Jeanette Medina pediatra de CESFAM número 1 Ramón Corbalán Melgarejo, a la fonoaudióloga Vanessa Quilempan de CESFAM Chuchunco; además de Enfermeras y TENS del CESFAM número 1 Ramón Corbalán Melgarejo y Chuchunco; pues sin su apoyo este trabajo no hubiese sido posible. También al estadístico Vicente Correa, que participó sin fines de lucro y sin cuya activa colaboración, no habría sido posible la ejecución del presente proyecto. Además, agradecemos a todas las familias y los niños que han participado voluntariamente en este trabajo y a quienes debemos el sentido de nuestra actividad asistencial y de investigación.

\section{Conflicto de intereses}

Los autores declaran no tener conflicto de intereses. 


\section{Referencias}

1. Diagnostic and Statistical Manual of Mental Disorders: DSM-5. 5th ed., American Psychiatric Association, 2013.

2. Fombonne E, Marcin C, Manero AC et al. Prevalence of Autism Spectrum Disorders in Guanajuato, Mexico: The Leon survey. J Autism Dev Disord. 2016;46(5):1669-85.

3. Zablotsky B, Black LI, Maenner MJ, et al. Estimated prevalence of autism and other developmental disabilities following questionnaire changes in the 2014 national health interview survey. Natl Health Stat Report. 2015;(87):1-20.

4. Elsabbagh M, Divan G, Koh YJ et al. Global Prevalence of Autism and Other Pervasive Developmental Disorders. Autism Res. 2012;5(3):160-79.

5. Davidovitch M, Hemo B, ManningCourtney P, et al. Prevalence and incidence of autism spectrum disorder in an Israeli population. J Autism Dev Disord. 2013;43(4):785-93.

6. Weintraub K. The prevalence puzzle: Autism counts. Nature. 2011;479(7371):22-4.

7. Daniel WW, Cross CL. Estimation. En: Daniel WW, Cross CL. Biostatistics: a foundation for analysis in the health sciences (10th edition). USA: Wiley, 2013;161-213.

8. INE: XVIII Censo nacional de población y VII de vivienda 2012.

9. Canal-Bedia R, García-Primo P, MartínCilleros MV et al. Modified checklist for autism in toddlers: Cross-cultural adaptation and validation in Spain. J Autism Dev Disord. 2011;41(10):1342-51.

10. Lord C, Rutter M, DiLavore PC, et al. PARTE I: Módulos 1 a 4 En: ADOS-2. Escala de Observación para el Diagnóstico del Autismo. Madrid: TEA Ediciones 2015;11-204.

11. Lord C, Luyster RJ, Gotham K, et al. PARTE II: Módulo T. En: ADOS-2. Escala de Observación para el Diagnóstico del Autismo. Madrid: TEA Ediciones 2015;213-353.

12. Coelho-Medeiros ME, Bronstein J, Aedo
K, et al. Validación del M-CHAT-R/F como instrumento de tamizaje para detección precoz en niños con trastorno del espectro autista. Rev Chil Pediatr. 2019;90(5):492-9.

13. Szklo M, Nieto F J. Epidemiologic issues in the interface with public health policy. En: Szklo M, Nieto F J. Epidemiology: beyond the basics (4th edition). Burlington, MA: Jones \& Bartlett Publishers 2018:437-78.

14. Baumer N, Spence SJ. Evaluation and Management of the Child With Autism Spectrum Disorder. Continuum (Minneap Minn) 2018;24(1):248-75.

15. Germain B, Eppinger MA, Mostofsky $\mathrm{SH}$, et al. Recent Advances in Understanding and Managing Autism Spectrum Disorders. J Child Neurol. 2015;30(14):1887-1920.

16. Fombonne E. Editorial: The rising prevalence of autism. J Child Psychol Psychiatry Allied Discip. 2018;59(7):71720.

17. Montiel-Nava C, Peña JA. Epidemiological findings of pervasive developmental disorders in a Venezuelan study. Autism. 2008;12(2):191-202.

18. Lejarraga $\mathrm{H}$, Menéndez $\mathrm{AM}$, Menzano E et al. Screening for developmental problems at primary care level: A field programme in San Isidro, Argentina. Paediatr Perinat Epidemiol. 2008;22(2):180-87.

19. Paula CS, Ribeiro SH, Fombonne E, et al. Brief report: Prevalence of pervasive developmental disorder in Brazil: A pilot study. J Autism Dev Disord. 2011:41(12);1738-42.

20. Fortea MS, Escandell MO, Castro JJ. Estimación de la prevalencia de los trastornos del espectro autista en Canarias. An Pediatr (Barc). 2013;79(6):352-59.

21. Ministerio de salud. Programa nacional de salud de la infancia con enfoque integral. Santiago, 2013. [Acceso: 20 Agosto 2020]. Disponible en: https:// www.minsal.cl/portal/url/item/ e2323d12579b8c79e0400101650124b6.pdf
22. Ministerio de Desarrollo Social y Familia. Encuesta de Caracterización Socioeconómica Nacional (CASEN) Santiago, 2011.

23. McPheeters M, Weitlauf A, Verhorn A et al. Screening for autism Spectrum Disorder in Young Children: A Systematic Evidence Review for the U.S. Preventive Services Task Force 2016;13-05185E(129):202.

24. Pasco G. The value of early intervention for children with autism. Paediatr Child Health. 2018;28(8):364-7.

25. García R, Irarrázabal M, Moyano A et al. Encuesta latinoamericana sobre las necesidades de los cuidadores de personas con trastornos del espectro autista. Libro de resúmenes. Rev Chil Psiquiatr Neurol Infanc Adolesc. 2016;27(3):57-8.

26. Prevalence of autism spectrum disorder among children aged 8 years - autism and developmental disabilities monitoring network, 11 sites, United States, 2010. MMWR Surveill Summ. 2014;63(2):1-21.

27. Johnson CP, Myers SM, Lipkin PH et al. Identification and evaluation of children with autism spectrum disorders. Pediatrics 2007;120(5):1183-215.

28. Robins DL, Fein D, Barton ML, et al. The Modified Checklist for Autism in Toddlers: An Initial Study Investigating the Early Detection of Autism and Pervasive Developmental Disorders. J Autism Dev Disord. 2001;31(2):131-44.

29. Robins DL. Screening for autism spectrum disorders in primary care settings. Autism. 2008;12(5):537-56.

30. Chlebowski C, Robins DL, Barton ML, et al. Large-scale use of the modified checklist for autism in low-risk toddlers. Pediatrics 2013;131(4).

31. Zwaigenbaum L, Bauman ML, Fein D et al. Early screening of autism spectrum disorder: Recommendations for practice and research. Pediatrics 2015;136:S41SS59.

32. Øien RA, Candpsych SS, Volkmar FR et al. Clinical features of children with autism who passed 18-month screening. Pediatrics 2018;141(6):e20173596. 